\title{
MIMO Equalization for Multi-Gbit/s Access Nodes Affected by Manufacturing Tolerances
}

\author{
L. Jacobs*, J. Bailleul*, P. Manfredi ${ }^{\dagger}$, M. Guenach* ${ }^{* \ddagger}$ D. Vande Ginste ${ }^{\dagger}$, and M. Moeneclaey* \\ * Department of Telecommunications and Information Processing, Ghent University, Ghent, Belgium \\ Email: \{lennert.jacobs,jelle.bailleul,marc.moeneclaey\}@ugent.be \\ $\dagger$ Department of Information Technology, Ghent University, Ghent, Belgium \\ Email: \{paolo.manfredi,dries.vande.ginste\}@ugent.be \\ $\ddagger$ Nokia Bell Labs, Antwerp, Belgium \\ Email: mamoun.guenach@nokia-bell-labs.com
}

\begin{abstract}
While the requirements for delivering high throughputs increase exponentially with every generation of access node hardware, the device cost is of primary concern. As a result, multiple-input multipleoutput (MIMO) equalization, which has been shown to facilitate multi-Gbit/s communication over low-cost parallel electrical interconnects, is emerging as an attractive high-speed interconnect solution for next-generation access nodes. Because of the high operating frequencies, however, the transfer functions of the on- and off-chip interconnects become highly susceptible to manufacturing tolerances (MTs); hence, the equalization filters must be adjusted to the specific channel realization to achieve optimal performance, which involves a high implementation and computational complexity. Considering that the MTs are usually limited, we propose a robust lowcomplexity transceiver consisting of a fixed MIMO linear pre-equalizer (which avoids the need for feeding back the channel state information to the transmitter), with either a fixed or adjustable MIMO decision-feedback equalizer (DFE). For a specific chip-to-chip interconnect operating at $75 \mathrm{Gbit} / \mathrm{s}$ per line and a $26 \mathrm{~dB}$ signal-to-noise ratio, we show that the resulting bit error rate does not exceed $10^{-12}$ for MTs up to $\mathbf{1 0 . 5 \%}$ (fixed DFE) and $\mathbf{1 7 . 7 \%}$ (adjustable DFE) of the nominal line width.
\end{abstract}

\section{INTRODUCTION}

Advances in access network last-mile technologies put increasingly higher demands on access nodes. This is reflected in the growing capabilities and capacities of internal components and, consequently, the interconnects linking those elements. Currently, most state-of-the-art transceivers apply single-input singleoutput (SISO) decision-feedback equalization (DFE) or Tomlinson-Hirashima precoding (THP), to deal with the inter symbol interference (ISI) caused by highfrequency attenuation [1], [2]. In addition, various techniques have been proposed to mitigate crosstalk (XT) from adjacent conductors [3]-[5]. Nonetheless, it was shown in [6] that employing multiple-input multiple-output (MIMO) equalization allows to significantly improve multi-Gbit/s communication over lowcost parallel electrical interconnects by exploiting the XT signals rather than suppressing them. Because of the high operating frequencies of the transceiver, however, the electrical interconnects become particularly prone to manufacturing tolerances (MTs), requiring that the equalization filters be adjusted to the specific channel realization to achieve optimal performance. To reduce the implementation complexity associated with adjustable equalizers, we propose a MIMO equalization scheme which generalizes the reduced-complexity approach for SISO interconnects in [7], where (part of) the equalization filters depend on the channel statistics rather than the actual channel. More specifically, by combining a fixed MIMO linear pre-equalizer at the transmitter with fixed or adjustable MIMO DFE at the receiver, complicated feedback of accurate channel information to the transmitter can be avoided. We show that the level of channel variability determines whether the fixed, hybrid (mixed fixed and adjustable), or fully adjustable equalization scheme is to be preferred for achieving a target error performance. In addition, we address the impact of the sampling phase, the operating rate of the pre-equalizers, and the number of equalization taps on the error performance.

\section{SYSTEM MODEL}

We consider the baseband transceiver from Fig. 1. $L$ real-valued spatially and temporally independent data streams $\left\{a^{(l)}(k)\right\}$ at symbol rate $1 / T$ per stream, with $1 \leq l \leq L$, are upsampled by a factor $N_{\mathrm{p}}$, and applied to a $L \times L$ MIMO pre-equalizer, operating at rate $1 / T_{\mathrm{p}}=N_{\mathrm{p}} / T$; the resulting equalizer transfer function is periodic with period $1 / T_{\mathrm{p}}$. The $L$ outputs of the pre-equalizer are each applied to a pulse shaping filter $h_{\mathrm{tr}}(t)$ at rate $1 / T_{\mathrm{p}}$ and transmitted on $L$ mutually coupled channels. The continuous-time signal $s^{(q)}(t)$ at the $q$-th output of the transmitter is given by

$s^{(q)}(t)=\sum_{p=1}^{L} \sum_{n, k} a^{(p)}(k) h_{\mathrm{p}}^{(q, p)}(n) h_{\mathrm{tr}}\left(t-k T-n T_{\mathrm{p}}\right)$,

where $h_{\mathrm{p}}^{(q, p)}(m)$ denotes the impulse response between the $p$-th input and the $q$-th output of the pre-equalizer. The channel matrix $\mathbf{H}_{\mathrm{ch}}(t)$ captures the impulse responses $h_{\mathrm{ch}}^{(l, q)}(t)$ between the $q$-th transmitter and the $l$-th receiver, with $1 \leq l, q \leq L$. The $L$ received signals, affected by channel dispersion and XT, are each filtered by an analog receiver filter $h_{\text {rec }}(t)$ and 


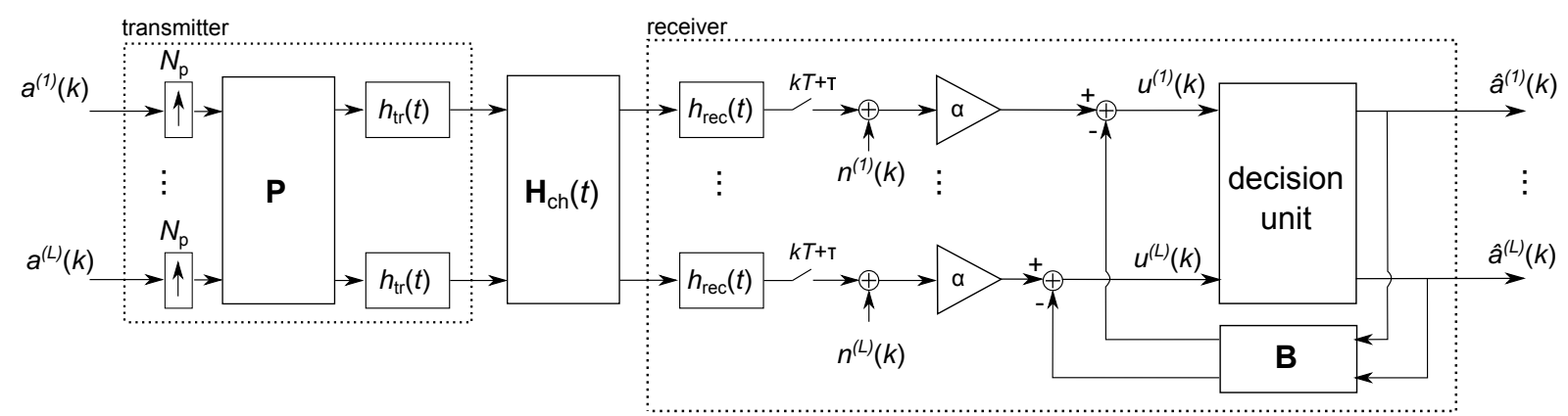

Figure 1. MIMO DFE scheme.

synchronously sampled at the symbol rate, at instants $k T+\tau$; the sampling delay $\tau$ can compensate for the delays introduced by the transmit filters, the channel, and the receive filters. The stationary noise at the receiver is represented by the samples $\left\{n^{(l)}(k)\right\}$, with $1 \leq l \leq L$. Finally, the decision variables $\left\{u^{(l)}(k)\right\}$, with $1 \leq l \leq L$, are obtained by scaling the received samples with a common factor $\alpha$ and by subtracting from the result the outputs of the $L \times L$ MIMO decision-feedback equalizer, which operates on past symbol decisions. Assuming that the latter decisions are correct, the decision variables are given by

$$
\begin{aligned}
& u^{(l)}(k)=\alpha \sum_{p=1}^{L} \sum_{m} h^{(l, p)}(m) a^{(p)}(k-m) \\
& +\alpha n^{(l)}(k)-\sum_{p=1}^{L} \sum_{m>0} h_{\mathrm{b}}^{(l, p)}(m) a^{(p)}(k-m),
\end{aligned}
$$

where $h_{\mathrm{b}}^{(l, p)}(m)$ denotes the strictly causal impulse response between the $p$-th input and the $l$-th output of the feedback equalizer, and $h^{(l, p)}(m)$ is defined as

$$
h^{(l, p)}(m)=\sum_{q=1}^{L} \sum_{n} g^{(l, q)}\left(m N_{\mathrm{p}}-n\right) h_{\mathrm{p}}^{(q, p)}(n)
$$

where the sequences $\left\{g^{(l, q)}(m)\right\}$, with $1 \leq l, q \leq L$, are obtained by sampling at instants $\left\{m T_{\mathrm{p}}+\tau\right\}$ the convolution of the impulse responses $h_{\mathrm{tr}}(t), h_{\mathrm{ch}}^{(l, q)}(t)$, and $h_{\text {rec }}(t)$. Ideally, in the absence of noise, ISI and XT, we should have $u^{(l)}(k)=a^{(l)}(k)$.

In practice, the equalizers are finite impulse response (FIR) filters with a limited number of taps. For the pre-equalizer, we assume $h_{\mathrm{p}}^{(q, p)}(m)=0$ for $m \notin\left[-L_{\mathrm{p}, 1}, L_{\mathrm{p}, 2}\right]$, yielding $L_{\mathrm{p}}=L_{\mathrm{p}, 1}+L_{\mathrm{p}, 2}+1$ taps per filter. At the receiver we assume $L_{\mathrm{b}}$ taps per feedback filter, such that $h_{\mathrm{b}}^{(l, p)}(m)=0$ for $m \notin \Psi_{\mathrm{b}}$, with $\Psi_{\mathrm{b}}=\left[1, L_{\mathrm{b}}\right]$. In order to enable convenient matrix notation, we introduce the $\left(L L_{\mathrm{p}}\right) \times L$ block matrix $\mathbf{P}$, comprising all pre-equalizer coefficients, as:

$$
\mathbf{P}=\left[\begin{array}{c}
\breve{\mathbf{P}}\left(-L_{\mathrm{p}, 1}\right) \\
\vdots \\
\breve{\mathbf{P}}\left(L_{\mathrm{p}, 2}\right)
\end{array}\right]
$$

where the $(q, p)$-th entry of the $L \times L$ matrix $\breve{\mathbf{P}}(m)$ is given by $h_{\mathrm{p}}^{(q, p)}(m)$. The samples $\left\{g^{(l, q)}(m)\right\}$ are included in the $L \times\left(L L_{\mathrm{p}}\right)$ channel block matrix $\mathbf{G}(m)$, which is defined as

$$
\mathbf{G}(m)=\left[\begin{array}{lll}
\breve{\mathbf{G}}_{m}\left(-L_{\mathrm{p}, 1}\right) & \ldots & \breve{\mathbf{G}}_{m}\left(L_{\mathrm{p}, 2}\right)
\end{array}\right],
$$

where the $(l, q)$-th entry of the $L \times L$ matrix $\breve{\mathbf{G}}_{m}(n)$ is given by $g^{(l, q)}\left(m N_{\mathrm{p}}-n\right)$. The sequences $\left\{g^{(l, q)}(m)\right\}$ are assumed to have limited time duration, i.e., $g^{(l, q)}(m)=0$ for $m \notin\left[-L_{g, 1}, L_{g, 2}\right]$, such that the matrices $\mathbf{G}(m)$ are nonzero only for $m \in \Psi_{\mathrm{G}}$, with $\Psi_{\mathrm{G}}=\left[-L_{\mathrm{G}, 1}, L_{\mathrm{G}, 2}\right], L_{\mathrm{G}, 1}=\left\lfloor\left(L_{g, 1}+L_{\mathrm{p}, 1}\right) / N_{\mathrm{p}}\right\rfloor$, and $L_{\mathrm{G}, 2}=\left\lfloor\left(L_{g, 2}+L_{\mathrm{p}, 2}\right) / N_{\mathrm{p}}\right\rfloor$. The $L \times\left(L L_{\mathrm{b}}\right)$ block matrix $\mathbf{B}=\left[\mathbf{B}(1), \ldots, \mathbf{B}\left(L_{\mathrm{b}}\right)\right]$ comprises all feedback filter taps, as the $(l, p)$-th entry of the $L \times L$ matrix $\mathbf{B}(m)$ is equal to $h_{\mathrm{b}}^{(l, p)}(m)$, for $m \in \Psi_{\mathrm{b}}$. Finally, by introducing the $L$-dimensional column vectors $\mathbf{a}(k), \mathbf{n}(k)$, and $\mathbf{u}(k)$, the $l$-th elements of which are given by $a^{(l)}(k), n^{(l)}(k)$, and $u^{(l)}(k)$, respectively, the vector of decision variables is given by

$$
\begin{aligned}
\mathbf{u}(k) & =\alpha\left[\sum_{m \in \Psi_{\mathrm{G}}} \mathbf{G}(m) \mathbf{P} \mathbf{a}(k-m)+\mathbf{n}(k)\right] \\
& -\sum_{m \in \Psi_{\mathrm{b}}} \mathbf{B}(m) \mathbf{a}(k-m) .
\end{aligned}
$$

\section{MMSE MIMO DFE}

Because of manufacturing tolerances on the electrical interconnect, the matrices $\mathbf{G}(m)$ from (5) are random, rather than deterministic. We assume the availability of $J$ realizations of $\left\{\mathbf{G}(m), m \in \Psi_{\mathrm{G}}\right\}$, which are denoted $\left\{\mathbf{G}_{j}(m), m \in \Psi_{\mathrm{G}}\right\}$, with $1 \leq j \leq$ $J$. Defining the error vector between the actual output $\mathbf{u}(k)$ and the target output $\mathbf{a}(k)$ as $\mathbf{e}(k)=\mathbf{u}(k)-\mathbf{a}(k)$, it follows from (6) that, for the $j$-th channel realization, $\mathbf{e}(k)$ can be written as

$$
\begin{aligned}
& \mathbf{e}(k)=\sum_{m \in \Psi_{\mathrm{b}}}\left(\alpha \mathbf{G}_{j}(m) \mathbf{P}-\mathbf{B}(m)\right) \mathbf{a}(k-m) \\
& +\alpha \mathbf{n}(k)+\sum_{m \in \Psi_{0}}\left(\alpha \mathbf{G}_{j}(m) \mathbf{P}-\delta_{m} \mathbf{I}_{L}\right) \mathbf{a}(k-m),
\end{aligned}
$$

where $\Psi_{0}=\Psi_{\mathrm{G}} \backslash \Psi_{\mathrm{b}}$. The equalization matrices $\mathbf{P}$ and $\mathbf{B}$ must be selected so as to keep the error 
$\mathbf{e}(k)$ small. More specifically, the minimum mean square error (MMSE) equalization filters minimize the average MSE, which is defined as:

$$
\mathrm{MSE}_{\mathrm{avg}} \triangleq \frac{E\left[\|\mathbf{e}(k)\|^{2}\right]}{E\left[\|\mathbf{a}(k)\|^{2}\right]}
$$

where the expectation in the numerator is taken over the data symbols, the noise, and the channel realizations. For simplicity of notation, we introduce the conditional MSE, conditioned on the channel realization $\mathbf{G}_{j}(m)$ and denoted by $\mathrm{MSE}_{j}$. In this way, (8) can be obtained as

$$
\mathrm{MSE}_{\mathrm{avg}}=\mathbb{E}_{j}\left[\mathrm{MSE}_{j}\right]=\frac{1}{J} \sum_{j=1}^{J} \mathrm{MSE}_{j},
$$

where we use the notation $\mathbb{E}_{j}[$.$] to denote averaging$ over the channel realizations. The conditional MSE follows from (7) as:

$$
\begin{aligned}
& \mathrm{MSE}_{j}=\frac{1}{L}\left[\sum_{m \in \Psi_{\mathrm{b}}}\left\|\alpha \mathbf{G}_{j}(m) \mathbf{P}-\mathbf{B}(m)\right\|^{2}\right. \\
& \left.+\sum_{m \in \Psi_{0}}\left\|\alpha \mathbf{G}_{j}(m) \mathbf{P}-\delta_{m} \mathbf{I}_{L}\right\|^{2}+\frac{\alpha^{2}}{\sigma_{a}^{2}} \operatorname{tr}\left(\mathbf{R}_{\mathbf{n}}\right)\right]
\end{aligned}
$$

where $\sigma_{a}^{2}=E\left[\left(a^{(p)}(k)\right)^{2}\right]$ is the symbol variance, and $\mathbf{R}_{\mathbf{n}} \triangleq E\left[\mathbf{n}(k) \mathbf{n}(k)^{\mathrm{T}}\right]$ is the $L \times L$ noise autocorrelation matrix. As the transmitter has limited power, the pre-equalization filters need to be selected such that the average transmitted energy per symbol does not exceed $E_{\mathrm{s}}$, i.e., the total transmitted energy per symbol interval is limited to $L E_{\mathrm{s}}$. It can be obtained from (1) that the latter energy constraint reduces to:

$$
\sigma_{a}^{2} \operatorname{tr}\left(\mathbf{P}^{\mathrm{T}} \mathbf{G}_{\mathrm{tr}} \mathbf{P}\right)=L E_{\mathrm{s}},
$$

where the elements of the $\left(L L_{\mathrm{p}}\right) \times\left(L L_{\mathrm{p}}\right)$ matrix $\mathbf{G}_{\mathrm{tr}}$ are given by

$$
\left(\mathbf{G}_{\mathrm{tr}}\right)_{n_{1}, n_{2}}=\int_{-\infty}^{+\infty} h_{\mathrm{tr}}(t) h_{\mathrm{tr}}\left(t+\left(n_{1}-n_{2}\right) T_{\mathrm{p}}\right) d t
$$

\section{A. Adjustable equalization scheme}

When the equalization filters in (10) are adjusted according to the specific channel realization $\mathbf{G}_{j}(m)$, the average MSE (9) is minimized by minimizing each term $\mathrm{MSE}_{j}$ separately.

For any given pre-equalization matrix $\mathbf{P}_{j}$ and scaling factor $\alpha_{j}$, the optimal feedback filters $\mathbf{B}_{\mathrm{a}, j}(m)$, with $m \in \Psi_{\mathrm{b}}$, that minimize $\mathrm{MSE}_{j}$ are readily obtained from (10):

$$
\mathbf{B}_{\mathrm{a}, j}(m)=\alpha_{j} \mathbf{G}_{j}(m) \mathbf{P}_{j}
$$

In this way, the conditional MSE (10) reduces to

$$
\begin{aligned}
\mathrm{MSE}_{j} & =\frac{1}{L}\left[\sum_{m \in \Psi_{0}}\left\|\alpha_{j} \mathbf{G}_{j}(m) \mathbf{P}_{j}-\delta_{m} \mathbf{I}_{L}\right\|^{2}\right. \\
& \left.+\frac{\alpha_{j}^{2}}{\sigma_{a}^{2}} \operatorname{tr}\left(\mathbf{R}_{\mathbf{n}}\right)\right] .
\end{aligned}
$$

The optimal MMSE pre-equalizer $\mathbf{P}_{\mathrm{a}, j}$ is obtained along with the optimal scaling factor $\alpha_{\mathrm{a}, j}$ by minimizing (14) under the energy constraint (11). The corresponding Lagrangian $\Lambda_{j}$ is given by

$$
\Lambda_{j}=\mathrm{MSE}_{j}+\lambda_{j} \frac{\sigma_{a}^{2} \operatorname{tr}\left(\mathbf{P}_{j}^{\mathrm{T}} \mathbf{G}_{\mathrm{tr}} \mathbf{P}_{j}\right)-L E_{\mathrm{s}}}{L \sigma_{a}^{2}},
$$

where $\lambda_{j}$ is the Lagrange multiplier and $\mathrm{MSE}_{j}$ is given by (14). $\mathbf{P}_{\mathrm{a}, j}$ is obtained as a function of $\lambda_{j}$ and $\alpha_{j}$ from minimizing (15) with respect to $\mathbf{P}_{j}$ :

$$
\mathbf{P}_{\mathrm{a}, j}=\frac{1}{\alpha_{j}} \mathbf{D}_{\mathrm{a}, j}^{-1} \mathbf{G}_{j}(0)^{\mathrm{T}},
$$

where

$$
\mathbf{D}_{\mathrm{a}, j} \triangleq \sum_{m \in \Psi_{0}} \mathbf{G}_{j}(m)^{\mathrm{T}} \mathbf{G}_{j}(m)+\frac{\lambda_{j}}{\alpha_{j}^{2}} \mathbf{G}_{\mathrm{tr}} .
$$

Finally, the optimal values $\lambda_{\mathrm{a}, j}$ and $\alpha_{\mathrm{a}, j}$ are obtained from the energy constraint (11) and the derivative of the Lagrangian (15) with respect to $\alpha_{j}$, which yields

$$
\lambda_{\mathrm{a}, j}=\alpha_{j}^{2} \frac{\operatorname{tr}\left(\mathbf{R}_{\mathbf{n}}\right)}{L E_{\mathrm{s}}},
$$

such that $\mathbf{D}_{\mathrm{a}, j}$ in (17) does not depend on $\alpha_{j}$ and

$$
\alpha_{\mathrm{a}, j}=\sqrt{\frac{\sigma_{a}^{2}}{L E_{\mathrm{s}}} \operatorname{tr}\left(\mathbf{G}_{j}(0) \mathbf{D}_{\mathrm{a}, j}^{-1} \mathbf{G}_{\mathrm{tr}} \mathbf{D}_{\mathrm{a}, j}^{-1} \mathbf{G}_{j}(0)^{\mathrm{T}}\right)} .
$$

\section{B. Fixed equalization scheme}

As a fully adjustable equalization scheme requires that all equalization taps must be tunable and accurate channel state information is required at both the transmitter and the receiver, its implementation complexity may be prohibitively large. Instead, here we consider a fixed equalization scheme that is independent of the actual channel realization. The matrix expressions for the equalization filters are obtained by minimizing the average MSE (9) over fixed $\alpha, \mathbf{P}$ and $\mathbf{B}$.

For a given pre-equalization matrix $\mathbf{P}$ and scaling factor $\alpha$, it is readily verified that the average MSE (9) is minimized by selecting the feedback filters for $m \in \Psi_{\mathrm{b}}$ as

$$
\mathbf{B}_{\mathrm{f}}(m)=\alpha \mathbb{E}_{j}\left[\mathbf{G}_{j}(m)\right] \mathbf{P} .
$$

In the case of fixed equalization filters, the Lagrangian $\Lambda$ is given by 


$$
\Lambda=\mathrm{MSE}_{\mathrm{avg}}+\lambda \frac{\sigma_{a}^{2} \operatorname{tr}\left(\mathbf{P}^{\mathrm{T}} \mathbf{G}_{\mathrm{tr}} \mathbf{P}\right)-L E_{\mathrm{s}}}{L \sigma_{a}^{2}},
$$

where $\mathrm{MSE}_{\mathrm{avg}}$ is given by (9). The MMSE preequalization filters are obtained as a function of $\alpha$ and $\lambda$ by minimizing (21) with respect to the preequalization matrix $\mathbf{P}$ using (20):

$$
\mathbf{P}_{\mathrm{f}}=\frac{1}{\alpha} \widetilde{\mathbf{P}}_{\mathrm{f}}
$$

where

$$
\widetilde{\mathbf{P}}_{\mathrm{f}}=\mathbf{D}_{\mathrm{f}}^{-1} \mathbb{E}_{j}\left[\mathbf{G}_{j}(0)^{\mathrm{T}}\right],
$$

and

$$
\begin{aligned}
& \mathbf{D}_{\mathrm{f}} \triangleq \sum_{m \in \Psi_{\mathrm{G}}} E_{j}\left[\mathbf{G}_{j}(m)^{\mathrm{T}} \mathbf{G}_{j}(m)\right] \\
& -\sum_{m \in \Psi_{\mathrm{b}}} \mathbb{E}_{j}\left[\mathbf{G}_{j}(m)^{\mathrm{T}}\right] \mathbb{E}_{j}\left[\mathbf{G}_{j}(m)\right]+\frac{\lambda}{\alpha^{2}} \mathbf{G}_{\mathrm{tr}} .
\end{aligned}
$$

Finally, the optimal values $\lambda_{\mathrm{f}}$ and $\alpha_{\mathrm{f}}$ can be obtained from the energy constraint (11) and the derivative of (21) with respect to $\alpha$, which yields

$$
\lambda_{\mathrm{f}}=\alpha^{2} \frac{\operatorname{tr}\left(\mathbf{R}_{\mathbf{n}}\right)}{L E_{\mathrm{s}}},
$$

and

$$
\alpha_{\mathrm{f}}=\sqrt{\frac{\sigma_{a}^{2}}{L E_{\mathrm{s}}} \operatorname{tr}\left(\widetilde{\mathbf{P}}_{\mathrm{f}}^{\mathrm{T}} \mathbf{G}_{\mathrm{tr}} \widetilde{\mathbf{P}}_{\mathrm{f}}\right)} .
$$

\section{Hybrid equalization scheme}

As it is expected that a fully fixed scheme is not resilient to large channel variability, we also investigate a hybrid approach, where an adjustable DFE at the receiver is combined with fixed pre-equalization, such that no channel state information needs to be fed back to the transmitter. As the feedback filters are adjustable, they are obtained in the same way as (13) by minimizing the conditional MSE (10):

$$
\mathbf{B}_{\mathrm{h}, j}(m)=\alpha \mathbf{G}_{j}(m) \mathbf{P} .
$$

The fixed pre-equalization matrix $\mathbf{P}$ and scaling factor $\alpha$ are then obtained by minimizing the Lagrangian (21), where $\mathrm{MSE}_{\mathrm{avg}}$ is the average MSE (9) and the feedback filters are given by (27):

$$
\mathbf{P}_{\mathrm{h}}=\frac{1}{\alpha_{\mathrm{h}}} \widetilde{\mathbf{P}}_{\mathrm{h}},
$$

with

$$
\widetilde{\mathbf{P}}_{\mathrm{h}}=\mathbf{D}_{\mathrm{h}}^{-1} \mathbb{E}_{j}\left[\mathbf{G}_{j}(0)^{\mathrm{T}}\right]
$$

and

$$
\mathbf{D}_{\mathrm{h}} \triangleq \sum_{m \in \Psi_{0}} \mathbb{E}_{j}\left[\mathbf{G}_{j}(m)^{\mathrm{T}} \mathbf{G}_{j}(m)\right]+\frac{\lambda}{\alpha^{2}} \mathbf{G}_{\mathrm{tr}}
$$

The optimal values $\lambda_{\mathrm{h}}$ and $\alpha_{\mathrm{h}}$ result from the energy constraint (11) and the derivative of (21) with respect to $\alpha$, which yields

$$
\lambda_{\mathrm{h}}=\alpha_{\mathrm{h}}^{2} \frac{\operatorname{tr}\left(\mathbf{R}_{\mathbf{n}}\right)}{L E_{\mathrm{s}}},
$$

and

$$
\alpha_{\mathrm{h}}=\sqrt{\frac{\sigma_{a}^{2}}{L E_{\mathrm{s}}} \operatorname{tr}\left(\widetilde{\mathbf{P}}_{\mathrm{h}}^{\mathrm{T}} \mathbf{G}_{\mathrm{tr}} \widetilde{\mathbf{P}}_{\mathrm{h}}\right)} .
$$

\section{NuMERICAL RESULTS}

We illustrate the MSE and BER performances of the above equalization strategies considering a chip-tochip interconnect consisting of 4 parallel $10-\mathrm{cm}$ long on-board microstrip lines, suitable for next-generation architectures of access network nodes. The nominal values of the geometrical and material parameters are as follows:

- width of signal conductors: $w=100 \mu \mathrm{m}$

- gap between two conductors: $s=80 \mu \mathrm{m}$

- thickness of signal conductor: $t_{k}=35 \mu \mathrm{m}$

- thickness of dielectric substrate: $h=500 \mu \mathrm{m}$

- conductivity of signal conductor: $\sigma=58 \mathrm{MS} / \mathrm{m}$

- relative permittivity of dielectric substrate: $\epsilon_{r}=4$

- loss tangent of dielectric substrate: $\tan \delta=0.02$

Because of MTs, the produced interconnects are not identical. We consider the line width $w$ as a Gaussian random variable, with a mean value equal to the nominal value. If $w$ changes, the gap between two conductors will change accordingly. The other parameters are assumed to be constant. In order to investigate the impact of the level of variability on the performance of the proposed equalization schemes, the standard deviation of $w$ takes on different values (ranging from $1 \%$ to $15 \%$ of the nominal value). We define the MT as three times this standard deviation, so that $99.7 \%$ of all signal conductors have a width within the range $(100 \mu \mathrm{m}-\mathrm{MT}, 100 \mu \mathrm{m}+\mathrm{MT})$; hence, a standard deviation of $3.3 \%$ corresponds to a $10 \%$ tolerance often assumed in consumer electronics. Obviously, the lower the standard deviation of the manufacturing uncertainty, the higher the production cost of the interconnects. We have generated 1000 realizations of $\mathbf{H}_{\mathrm{ch}}(f)$ with $|f|<100 \mathrm{GHz}$, using the polynomial chaos-based approach outlined in [8], [9].

The channel delay of the $l$-th direct channel is defined as the position of the peak of the convolution of the transmit pulse, the channel impulse response $h_{\mathrm{ch}}^{(l, l)}(t)$, and the receive filter. With $\tau_{0}$ being the largest channel delay of all direct channels, the sampling delay $\tau$ is selected as $\tau=\tau_{0}+\Delta_{\tau}$, where $\Delta_{\tau}$ is the sampling phase; when $\Delta_{\tau}=0$, the impulse response with largest delay is always sampled at its peak. In Fig. 2 , the average sampled impulse response $\mathbb{E}\left[g^{(1,1)}(m)\right]$ is shown for a rate $1 / T=75$ Gbaud and $\Delta_{\tau}=0$. The transmit and receive filters are assumed to be unitenergy square-root raised cosine filters having a $3 \mathrm{~dB}$ bandwidth of $1 / 2 T$ and a roll-off factor $\beta=0.3$. 


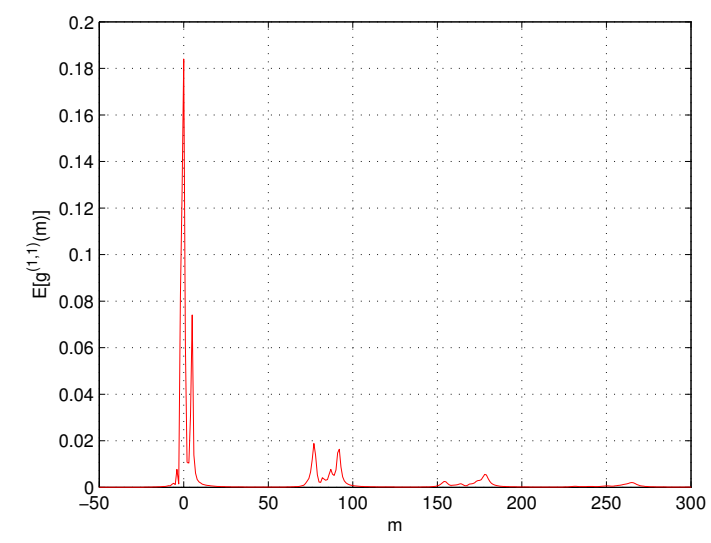

Figure 2. Average sampled impulse response $\mathbb{E}\left[g^{(1,1)}(m)\right]$ after time synchronization $\left(1 / T=75\right.$ Gbaud, $\left.N_{\mathrm{p}}=1\right)$.

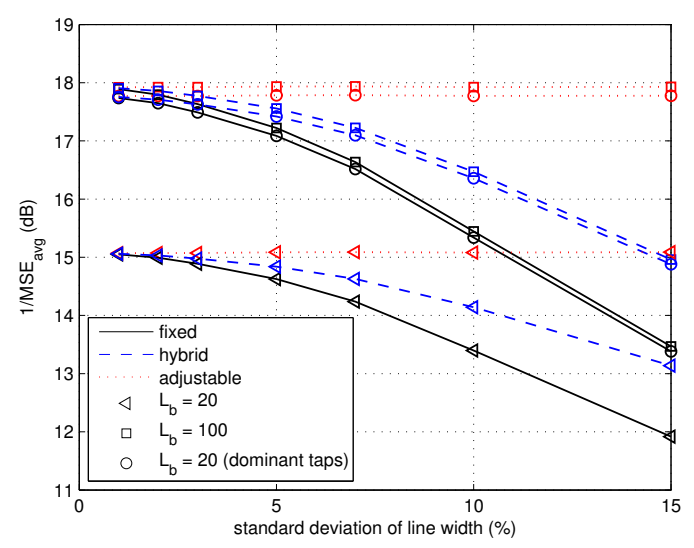

Figure 3. $1 / \mathrm{MSE}_{\mathrm{avg}}$ for MIMO equalization with $L_{\mathrm{p}, 1}=15$ and $L_{\mathrm{p}, 2}=5\left(N_{p}=1, \Delta_{\tau}=0\right)$.

Fig. 3 shows the inverse of the average MSE of the adjustable, fixed, and hybrid equalization schemes proposed in Section III versus the standard deviation of the random line width (expressed as a percentage of the nominal value). The results are averaged over the set of 1000 channel realizations. We assume a zero sampling phase $\Delta_{\tau}=0$, a symbol rate of $1 / T=75$ Gbaud, and a signal-to-noise ratio of $E_{\mathrm{s}} / N_{0}=26 \mathrm{~dB}$, with $N_{0} / 2$ being the variance of the i.i.d. Gaussian noise samples. The pre-equalization filters operate at the symbol rate (i.e., $N_{\mathrm{p}}=1$ ), and have $L_{\mathrm{p}, 1}=15$ anti-causal and $L_{\mathrm{p}, 2}=5$ causal taps. The feedback filters have either $L_{\mathrm{b}}=20$ or $L_{\mathrm{b}}=100$ coefficients; the former scheme can mitigate ISI from the main peak of the channel impulse response only, whereas the latter can also deal with ISI from the dominant reflection. Despite the significant MSE performance improvement that is observed from Fig. 3, increasing the number of feedback equalization taps from 20 to 100 may prohibitively increase the receiver complexity. Therefore, we exploit the observation that the dominant feedback taps correspond to the locations of the main pulse and the first reflection whereas the

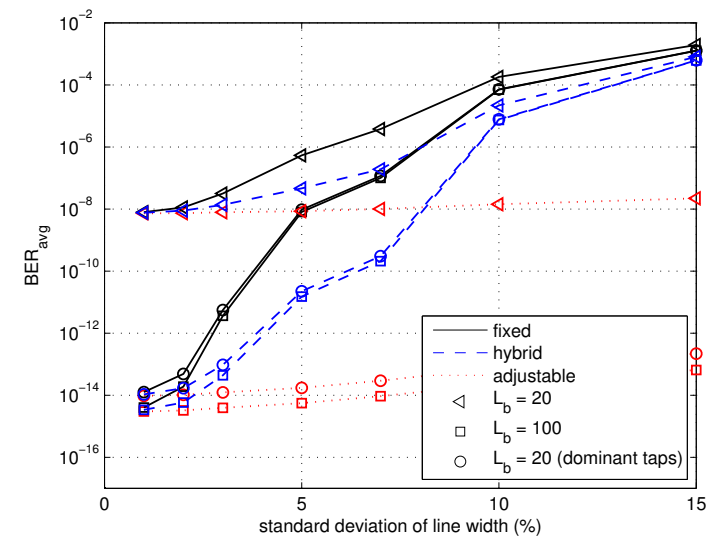

Figure 4. Average BER for MIMO equalization with $L_{\mathrm{p}, 1}=15$ and $L_{\mathrm{p}, 2}=5\left(N_{p}=1, \Delta_{\tau}=0\right)$.

remaining taps are very small. It follows from the figure that keeping from the 100 feedback taps only the 20 dominant taps with the largest magnitude, and setting to zero the remaining feedback taps results in a relatively small performance loss. Furthermore, the fully adjustable equalization schemes maintain a nearly constant average MSE irrespective of the level of variability, and largely outperform the fixed and hybrid schemes when the variability is very high. The corresponding average BERs, obtained using the semianalytical method from [10], are shown in Fig. 4. Assuming a 2-PAM constellation, a bit rate of $R_{\mathrm{b}}=75$ Gbit/s per channel is achieved. Obviously, a short feedback equalizer, that can only mitigate ISI from the main peak of the channel impulse response, is unable to meet a target BER of $10^{-12}$. When also addressing the ISI from the dominant reflection by keeping the 20 dominant taps of the 100-tap feedback equalizers, the target BER is achieved irrespective of the variability for the fully adjustable scheme, whereas for the fixed and the hybrid schemes the standard deviation of the line width must be lower than about $2.6 \%$ and $3.8 \%$, respectively; this corresponds to manufacturing tolerances of $3 \times 2.6=7.8 \%$ and $3 \times 3.8$ $=11.4 \%$, respectively.

In order to further improve the performance of the above MIMO equalization scheme with 20 dominant feedback taps, we consider increasing the operating rate of the pre-equalizer and optimizing the sampling phase, where $\Delta_{\tau}$ is in the range $\left[-T_{\mathrm{p}} / 2, T_{\mathrm{p}} / 2\right]$ and irrespective of the channel realization. From Fig. 5, it follows that optimizing the sampling phase (for $N_{\mathrm{p}}=1$ ) results in a relatively small improvement of the MSE performance of about $0.2 \mathrm{~dB}$, whereas increasing the operating rate of the pre-equalizer by a factor 2 (while keeping the number of filter taps constant) yields an improvement of up to $0.7 \mathrm{~dB}$. Note that, for $N_{\mathrm{p}}=2$, we consider the results for $\Delta_{\tau}=0$ only, because optimizing the sampling phase in the case of a pre-equalizer with increased operating 


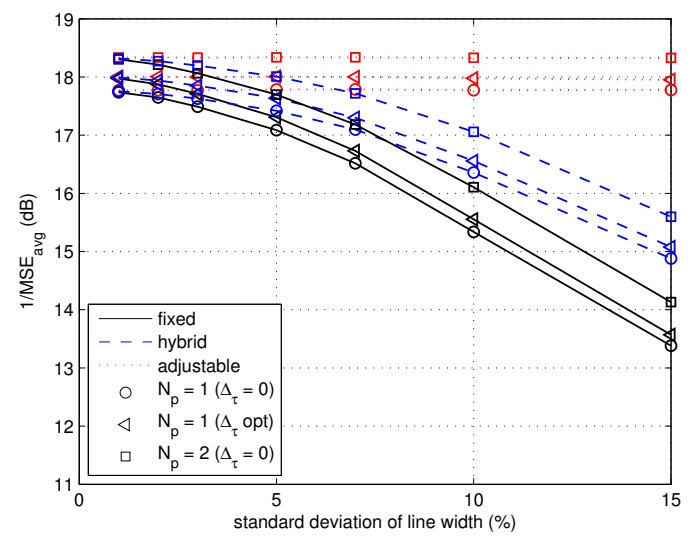

Figure 5. Impact of optimizing the sampling phase $\Delta_{\tau}$ and increasing the operating rate of the pre-equalizer on $1 / \mathrm{MSE}_{\mathrm{avg}}$.

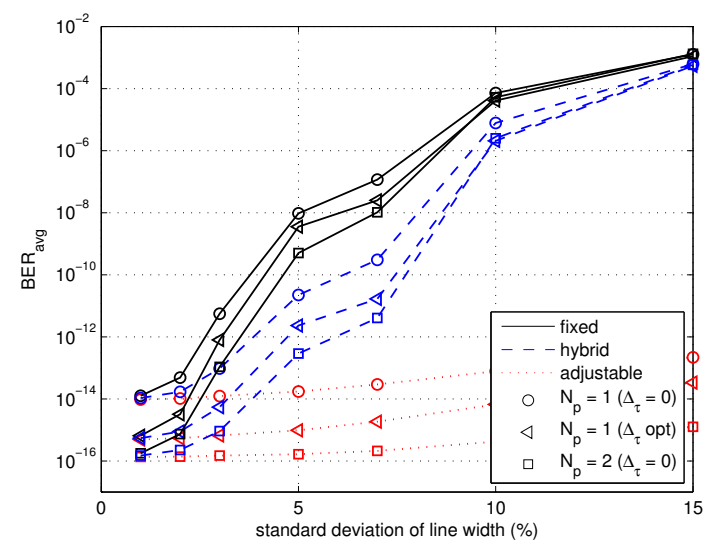

Figure 6. Impact of optimizing the sampling phase $\Delta_{\tau}$ and increasing the operating rate of the pre-equalizer on the average BER.

rate, was shown to have negligible impact on the error performance. The corresponding BER results from Fig. 6 illustrate that, for a target BER of $10^{-12}$, the maximum allowable standard deviation of the line width increases from $2.6 \%$ to $3.0 \%$ for the fixed scheme with $N_{\mathrm{p}}=1$ by optimizing the sampling phase and to about $3.5 \%$ by doubling the operating rate of the preequalizer. For the hybrid scheme, however, optimizing the sampling phase for $N_{\mathrm{p}}=1$ and doubling the operating rate increases the maximum allowable standard deviation from $3.8 \%$ to $4.7 \%$ and $5.9 \%$, respectively. Hence, with $N_{\mathrm{p}}=2$, fixed and hybrid equalization schemes can deal with manufacturing tolerances up to $3 \times 3.5=10.5 \%$ and $3 \times 5.9=17.7 \%$, respectively. Furthermore, irrespective of the variability, the fully adjustable equalization schemes achieve BERs below the target BER, which allows to reduce the number of equalization taps. However, a larger number of fixed pre-equalizer taps is generally preferred over an adjustable pre-equalizer with fewer filter taps, because the latter requires channel information to be fed back from the receiver.

\section{Conclusions}

In order to deal with channel variability in nextgeneration multi-Gbit/s access nodes, we proposed a general MIMO transceiver scheme where (part of) the equalization filters depend on the channel statistics rather than the actual channel realization. By considering fixed linear MIMO pre-equalization combined with fixed or adjustable MIMO DFE, complicated feedback of the channel information to the transmitter can be avoided. Both fixed and hybrid equalization schemes are shown to be able to deal with relatively large levels of variability.

\section{ACKNOWLEDGEMENTS}

Part of this research has been funded by the Interuniversity Attraction Poles Programme initiated by the Belgian Science Policy Office. The authors acknowledge the support of Bartek Kozicki from Nokia Bell Labs in providing inputs and review for this work.

\section{REFERENCES}

[1] H. Kimura, P. Aziz, T. Jing, A. Sinha, S. Kotagiri, R. Narayan, H. Gao, P. Jing, G. Hom, A. Liang, E. Zhang, A. Kadkol, R. Kothari, G. Chan, Y. Sun, B. Ge, J. Zeng, K. Ling, M. Wang, A. Malipatil, L. Li, C. Abel, and F. Zhong, "A $28 \mathrm{~Gb} / \mathrm{s} 560$ $\mathrm{mW}$ multi-standard SerDes with single-stage analog front-end and 14-tap decision feedback equalizer in $28 \mathrm{~nm}$ CMOS," IEEE J. Solid-State Circuits, vol. 49, no. 12, pp. 3091-3103, Dec 2014.

[2] Y. Iijima and Y. Yuminaka, "Double-rate equalization using tomlinson-harashima precoding for multi-valued data transmission," in IEEE 46th Int. Symp. on Multiple-Valued Logic (ISMVL), May 2016, pp. 66-71.

[3] K.-D. Hwang and L.-S. Kim, "A 6.5-Gb/s 1-mW/Gb/s/CH simple capacitive crosstalk compensator in a $130-n m$ process," IEEE Trans. Circuits Syst. II: Express Briefs, vol. 60, no. 6, pp. 302-306, June 2013.

[4] P. Amleshi and C. Gao, "NEXT and FEXT characteristics and suppressions in dense 25Gbps+ backplane vias," in IEEE Int. Symp. on Electromagnetic Compatibility (EMC), Aug 2014, pp. 979-985.

[5] D. Shilpa and B. Uma, "A wavelet technique to minimize offchip interconnect crosstalk," in Int. Conf. on Emerging Trends in Communication, Control, Signal Processing Computing Applications (C2SPCA), Oct 2013, pp. 1-5.

[6] L. Jacobs, M. Guenach, and M. Moeneclaey, "Linear MIMO equalization for high-speed chip-to-chip communication," in IEEE International Conference on Communications (ICC), June 2015, pp. 4978-4983.

[7] J. Bailleul, L. Jacobs, P. Manfredi, D. V. Ginste, and M. Moeneclaey, "MMSE equalization of multi-gb/s chip-tochip interconnects with M-PAM signaling affected by manufacturing tolerances," in IEEE Symposium on Communications and Vehicular Technologies (SCVT), Nov. 2016, pp. 1-6.

[8] P. Manfredi, D. Vande Ginste, D. De Zutter, and F. G. Canavero, "Generalized decoupled polynomial chaos for nonlinear circuits with many random parameters," IEEE Microw. Compon. Lett., vol. 25, no. 8, pp. 505-507, Aug 2015.

[9] D. Vande Ginste, D. De Zutter, D. Deschrijver, T. Dhaene, P. Manfredi, and F. Canavero, "Stochastic modeling-based variability analysis of on-chip interconnects," IEEE Trans. Compon. Packag. Manuf. Technol., vol. 2, no. 7, pp. 11821192, July 2012.

[10] M. Guenach, L. Jacobs, B. Kozicki, and M. Moeneclaey, "Performance analysis of pre-equalized multilevel partial response modulation for high-speed electrical interconnects," Computers and Electrical Engineering, vol. 58, pp. 30 - 48, 2017. 